\title{
Experimental Investigation on Flow-Induced Vibration of Fuel Rods in Supercritical Water Loop
}

\author{
Licun Wu, Daogang Lu, and Yu Liu \\ Nuclear Science and Engineering, North China Electric Power University, Beijing 102206, China \\ Correspondence should be addressed to Licun Wu; wlc121@126.com
}

Received 10 November 2013; Revised 15 January 2014; Accepted 15 January 2014; Published 24 February 2014

Academic Editor: Jiejin Cai

Copyright (C) 2014 Licun Wu et al. This is an open access article distributed under the Creative Commons Attribution License, which permits unrestricted use, distribution, and reproduction in any medium, provided the original work is properly cited.

\begin{abstract}
The supercritical water-cooled reactor (SCWR) is one of the most promising Generation IV reactors. In order to make the fuel qualification test for SCWR, a research plan is proposed to test a small scale fuel assembly in a supercritical water loop. To ensure the structure safety of fuel assembly in the loop, a flow-induced vibration experiment was carried out to investigate the vibration behavior of fuel rods, especially the vibration caused by leakage flow. From the experiment result, it can be found that: the vibration of rods is mainly caused by turbulence when flow rate is low. However, the effects of leakage flow become obvious as flow rate increases, which could changes the distribution of vibrational energy in spectrum, increasing the vibrational energy in highfrequency band. That is detrimental to the structure safety of fuel rods. Therefore, it is more reasonable to improve the design by using the spacers with blind hole, which can eliminate the leakage flow, to assemble the fuel rods in supercritical water loop. On the other hand, the experimental result could provide a benchmark for the theoretical studies to validate the applicability of boundary condition set for the leakage-flow-induced vibration.
\end{abstract}

\section{Introduction}

The supercritical water reactor is a Generation IV reactor concept which uses supercritical water as the working fluid. The high thermodynamic efficiency and plant simplification make SCWR attractive for consideration as a promising advanced nuclear system. In order to develop a viable design for the core, accurately estimate the heat transfer coefficient, and develop materials for the fuel and core structure that will be sufficiently corrosion-resistant to withstand SCWR conditions, a research plan, which is made by the steering committee of the SCWR system in the Generation IV International Forum, is proposed to test a small scale fuel assembly in a supercritical water loop in the LVR-15 research reactor, which is located in Nuclear Research Institute in Rez, Czech Republic. This water cooled reactor with an enough core height enables replacing one of its assemblies with a pressure tube containing four fuel rods, as Figure 1 shows. These four fuel rods can reach a fissile power of more than $50 \mathrm{~kW}$. A recuperator inside the pressure tube, situated right above the fuel rods, is boosting the feed-water temperature of $300^{\circ} \mathrm{C}$ to typical evaporator conditions. A cooler in the top section of the pressure tube acts as the heat sink to remove the fissile and gamma power. The pressure tube will be connected with coolant pumps and safety and auxiliary systems, forming the supercritical water loop to simulate a supercritical water environment at the fuel assembly while the rest of the core operates at ambient pressure (Figure 2). Before carrying out the project, a series of safety analyses on the small scale fuel assembly will be made, including the experimental investigation on the flow-induced vibration of fuel assembly, which is presented in this paper.

In general, there is a tiny clearance between the fuel rod and the spacer to make charging easier, and the wear between the fuel rods and spacer will make the clearance become larger over time. Consequently, the fuel rods would vibrate in case of coolant flowing through the narrow clearance. This phenomenon is called leakage-flow-induced vibration, which has been studied by many researchers (Blevins [1]; Hobson [2]; Chen [3]). Païdoussis [4] wrote a review to introduce some serious accidents in the nuclear reactors caused by it. 


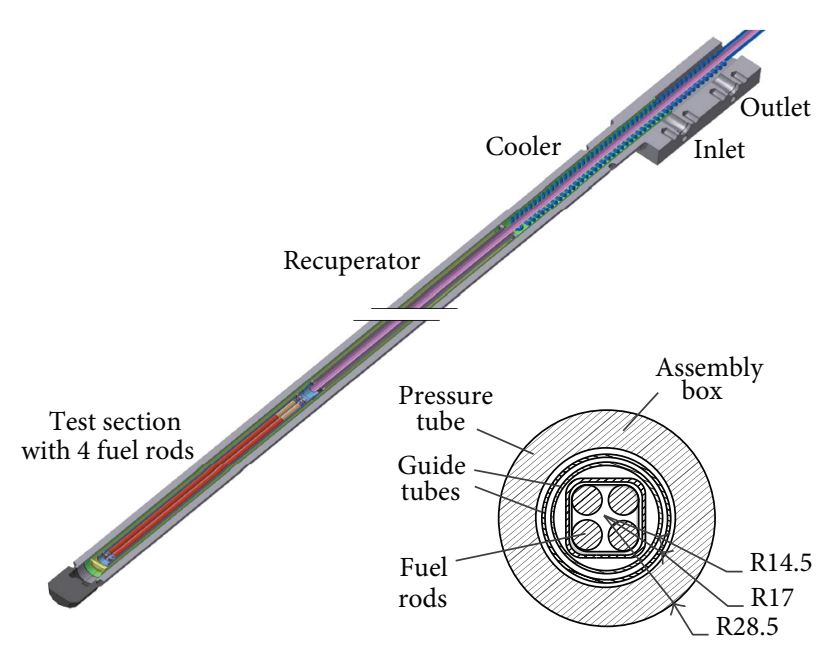

FIGURE 1: Concept of the active channel for SCWR fuel qualification.

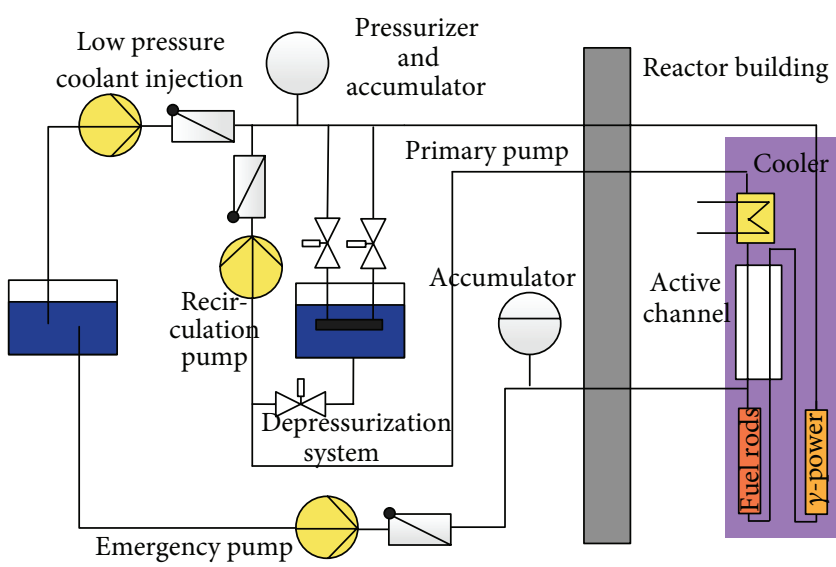

Figure 2: Schematic diagram of the primary loop and of the safety system for the SCWR fuel qualification test.

Mateescu and Paidoussis [5] researched the unsteady potential flow in a narrow annular passage, which is formed by a motionless rigid duct and an oscillating rigid centerbody, both of axially variable cross-sections. The fluid dynamic force exerted on the center-body was obtained on the basis of unsteady potential flow theory. It was concluded that the stability of center-body decreases as the pivot of rotation is shifted towards the downstream end of the centerbody, and a divergent annular passage has a destabilizing effect. Arai and Tajima [6] studied a similar problem by using a different approach.

Li et al. [7] developed a numerical method to analyze the flutter instability of the flow vibration of the inner cylinder. Meanwhile, an experiment on the annular leakage-flowinduced vibration was carried out, and a critical flow rate of the flutter instability was obtained for several annular leakage-flow systems with different passage increment ratios as well as the eccentricities. They found that a divergent annular passage may cause a negative fluid damping, so the flutter instability will happen when the flow rate is larger than the critical flow rate.

Langthjem et al. [8] investigated the linear stability of a flexible, cylindrical rod exposed in annular leakage flow. It is found that when flow speed reaches a certain critical value, simply supported rods may become unstable by either flutter or divergence.

Fujita et al. [9] investigated the dynamical behavior of an axisymmetric elastic beam subjected to axial leakage flow. By making complex eigenvalue analysis, the variation of the dynamic behavior during pre- and postinstability is researched with respect to increasing axial leakage-flow velocity. The relationships between the unstable phenomena and axial flow velocities are clarified for the transition from the lower mode to higher mode.

Since the study on leakage-flow-induced vibration of the fuel rods has not been reported previously, and the stability associated with the leakage flow mechanism strongly depends on the detailed geometries and fixed mode of the structure, it is necessary to perform an experiment to investigate the effects of leakage flow on the vibration of the small scale fuel rods in supercritical water loop. The present research is an extension of previous studies and aims at investigating the vibration characteristics of fuel rods under the influence of leakage flow. On one hand, the experimental results could provide a reference for optimal design of the fuel assembly in supercritical water loop. On the other hand, it could be treated as the benchmark to validate the availability of mathematical model built for the leakage-flow-induced vibration.

\section{Theoretical Models}

Consider a circular fuel rod immersed in the coolant flowing at velocity $U$ parallel to the $z$ axis (Figure 3 ). The fuel rod has flexural rigidity $E I$, linear density (mass per unit length) $m$, and total length $l$. All motions of the fuel rod are assumed to be confined in the $y$ - $z$ plane. According to the studies of Chen [3], the equation of motion is written as

$$
\begin{gathered}
E I \frac{\partial^{4} u}{\partial z^{4}}+\eta I \frac{\partial^{5} u}{\partial t \partial z^{4}}+m_{a} U^{2} \frac{\partial^{2} u}{\partial z^{2}}-\gamma T_{0} \frac{\partial^{2} u}{\partial z^{2}} \\
-\frac{1}{2} C_{T} \frac{m_{a} U^{2}}{D}\left[\left(1-\frac{1}{2} \gamma\right) l-z\right] \frac{\partial^{2} u}{\partial z^{2}} \\
-\frac{1}{2}(1-\gamma) C_{T}^{\prime} m_{a} U^{2} \frac{\partial^{2} u}{\partial z^{2}}+2 m_{a} U \frac{\partial^{2} u}{\partial z \partial t} \\
+\frac{1}{2} C_{N} \frac{m_{a} U^{2}}{D} \frac{\partial u}{\partial z}+\frac{1}{2} C_{N} \frac{m_{a} U}{D} \frac{\partial u}{\partial t} \\
+C_{V} \frac{\partial u}{\partial t}+\left(m+m_{a}\right) \frac{\partial^{2} u}{\partial t^{2}}=g(x, t),
\end{gathered}
$$

where $u$ is the displacement of fuel rod, $E$ is the modulus of elasticity, $m_{a}$ is the added mass, $T_{0}$ is the initial axial tension, $C_{T}^{\prime}$ is the form drag coefficient at the free end, $C_{V}$ is the effective viscous damping coefficient, $\gamma=1$ if the downstream end is supported so the displacement is zero, and $\gamma=0$ if it is 
TABLE 1: Natural frequency of the fuel rod.

\begin{tabular}{lcccc}
\hline Pellet material & First order & Second order & Third order & Fourth order \\
\hline Uranium dioxide & $38.1436 \mathrm{~Hz}$ & $38.1579 \mathrm{~Hz}$ & $111.947 \mathrm{~Hz}$ & $111.966 \mathrm{~Hz}$ \\
Lead & $37.738 \mathrm{~Hz}$ & $37.752 \mathrm{~Hz}$ & $110.76 \mathrm{~Hz}$ & $110.78 \mathrm{~Hz}$ \\
\hline
\end{tabular}

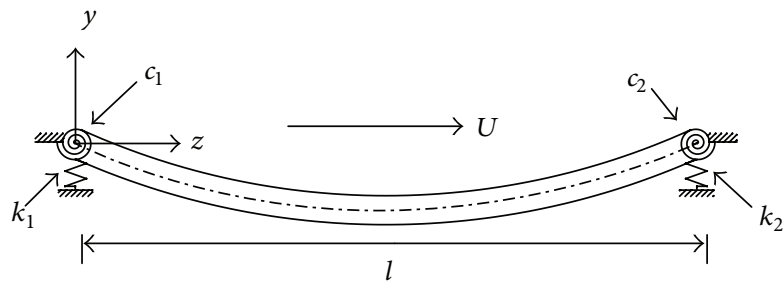

Figure 3: Schematic diagram of the fuel rod in axial flow.

unsupported or elastically supported. $C_{T}$ is equal to $C_{N}$ and defined as

$$
C_{N}=C_{T}=\frac{4 C_{f}}{\pi C_{m}}
$$

where $C_{f}$ is the drag coefficient due to shear forces and $C_{m}$ is added mass coefficient.

The appropriate boundary conditions associated with the equation of motion are as follows.

For $z=0$,

$$
k_{1} u+E I \frac{\partial^{3} u}{\partial z^{3}}=0, \quad c_{1} \frac{\partial u}{\partial z}-E I \frac{\partial^{2} u}{\partial z^{2}}=0
$$

and for $z=l$,

$$
k_{2} u-E I \frac{\partial^{3} u}{\partial z^{3}}=0, \quad c_{2} \frac{\partial u}{\partial z}+E I \frac{\partial^{2} u}{\partial z^{2}}=0
$$

where $k_{1}$ and $k_{2}$ are displacement spring constants and $c_{1}$ and $c_{2}$ are torsional spring constants (see Figure 3 ).

Unfortunately, (1) associated with boundary conditions (3) and (4) can only be used for the studies about linear vibration of the fuel rod. Since the boundary condition with leakage flow is nonlinear and complex, (3) and (4) are inappropriate as the boundary conditions for the calculation about leakage-flow-induced vibration of the fuel rod. On the other hand, since the boundary conditions are not taken into account during (1) derivation process, (1) is still applicable for calculation of leakage-flow-induced vibration. Therefore, only a series of appropriate boundary conditions are needed for (1) to accurately calculate the vibration behavior of fuel rod caused by leakage flow, and the experimental result in this paper could provide a benchmark to validate the availability of boundary conditions.

\section{Details of Experiment}

3.1. Experimental Setup. The experimental facility is shown in Figure 4. The flow-induced vibration test section consists of two parts: (1) a visualized channel made of acrylic with

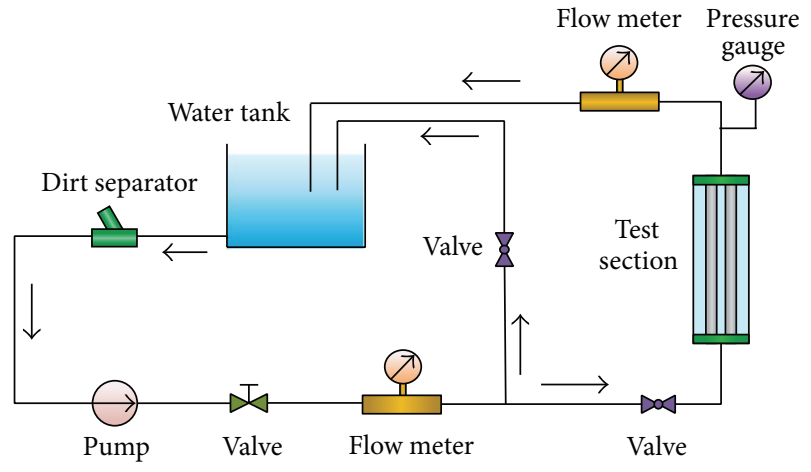

FIGURE 4: Schematic diagram of experimental loop.

TABLE 2: Experimental conditions and geometry parameters of the test section.

\begin{tabular}{lc}
\hline Temperature of water & $25^{\circ} \mathrm{C}$ \\
Operational pressure & $0.1 \mathrm{Mpa}$ \\
Size of channel & $20 \mathrm{~mm} \times 20 \mathrm{~mm} \times 725 \mathrm{~mm}$ \\
Diameter of fuel rod & $8 \mathrm{~mm}$ \\
Length of fuel rod & $710 \mathrm{~mm}$ \\
\hline
\end{tabular}

water flowing axially through it and (2) a small scale fuel assembly. The fuel assembly in the experiment has the same geometry parameters as that in supercritical water loop. The cladding is made of stainless steel 316L, which is the same as the material used in supercritical water loop. The material of pellet in the experiment is lead, whose density $\left(11.3 \mathrm{~g} / \mathrm{cm}^{3}\right)$ is similar to the density of uranium dioxide $\left(10.96 \mathrm{~g} / \mathrm{cm}^{3}\right)$. In order to demonstrate the rationality of using lead as a replacement for uranium dioxide, the modal analysis was made for the fuel rod. From the results of modal analysis listed in Table 1, it is easy to see there is little difference between the natural frequencies of fuel rods with two kinds of pellets made of uranium dioxide and lead, respectively. Since the vibration behavior of fuel rods is strongly dependent on their natural frequencies, it is proper to use lead as a replacement for uranium dioxide.

The bottom ends of the rods are fully constrained. Meanwhile, there is a $0.2 \mathrm{~mm}$ clearance between the top end of the rod and the locating hole. The displacements at midpoint of fuel rod are detected by a laser displacement sensor. The geometry parameters of the test section and experimental conditions are listed in Table 2. In addition, the assembly drawing and photo of the test section are shown in Figure 5.

In order to investigate the effects of leakage flow on the characteristics of flow-induced vibration, the effect of two different spacers, which is used to constrain the top end of the rod, is researched, respectively. Figure 6(a) illustrates the 


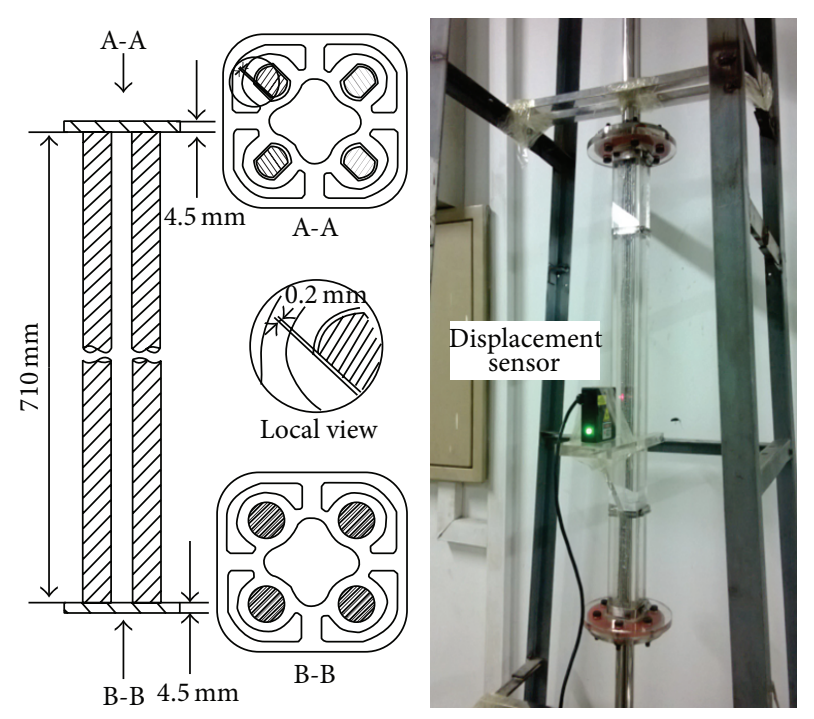

FIGURE 5: The collocation and geometry of the fuel assembly and test section.

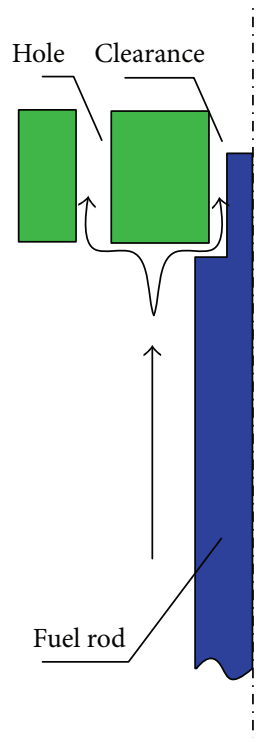

(a)

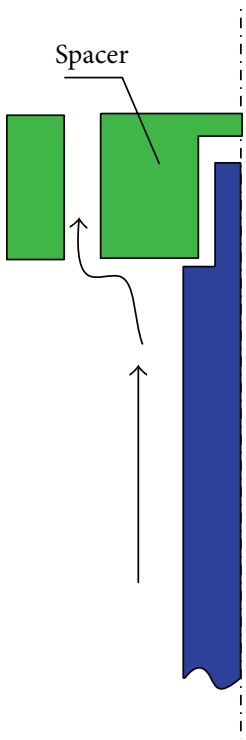

(b)
FIGURE 6: Schematic diagram of two types of spacers.

top end of fuel rod is constrained by the spacer with through holes, meaning there is a leakage flow across the clearance. This case is called "through hole case." In Figure 6(b), since the top end of fuel rod is constrained by the spacer with blind holes, there is no occurrence of leakage flow. This case is called "blind hole case."

3.2. Measuring Instrument Calibration. For the small size of channel, the contact measurement would have a significant influence on the flow field in the channel. Thus, the laser displacement sensor is used in the experiment to perform the noncontact measuring without influence on the flow field. It employs triangulation measurement principles whereby it projects a beam of visible laser light that creates a spot on a target surface. Reflected light from the surface is viewed from an angle by a digital camera inside the sensor, and the target's displacement is computed from the image pixel data.

However, in the process of measuring the vibration of fuel rod fixed in a channel with room-temperature water flowing inside, the refraction caused by acrylic, water, and air would lead to the measurement deviation. For accurately measuring the vibration of the fuel rods, it is necessary to correct the deviation of measurement caused by refraction before carrying out the flow-induced vibration (FIV) experiment.

Generally, the measuring instrument calibration can be performed by refraction theoretical analysis for laser displacement sensor. However, since the channel in practice cannot be made evenly by the acrylic material, the nonuniformity of channel could lead to the refraction occurring not only on the surface but also inside the channel, and this phenomenon is difficult to predict in theory. Thus, a device is employed to correct the measurement deviation caused by refraction. It is composed of a micrometer and a container made of acrylic, as Figure 7 shows.

The thickness of the container $d_{1}$ is the same as the thickness of channel $d_{1}^{\prime}$ in FIV experiment; meanwhile, the distance from the micrometer to the inner face of the container $d_{2}$ is equal to that from fuel rod to the inner wall of the channel $d_{2}^{\prime}$. Therefore, the measured deviation caused by refraction is the same when the sensor is used to measure the movements of the fuel rods and micrometer, respectively. When the top end of micrometer moves forward to a little distance $\Delta x$, a corresponding measured value $\Delta y$ can be obtained by the laser displacement sensor. The difference between $\Delta x$ and $\Delta y$ is the measured deviation caused by laser refraction. Several movements would be made to get two sets of data, $\Delta x_{n}(n=1,2,3,4 \ldots)$ and $\Delta y_{n}(n=1,2,3,4 \ldots)$. The calibration curve for laser displacement sensor could be obtained by a linear fitting with the two sets of data, as Figure 8 shows. The equation of the curve is written as

$$
y=0.72582 x-17.90196,
$$

where $x$ is the actual position of the micrometer and $y$ is the measured position.

Assuming the actual position is $x_{1}$, the measured position is $y_{1}$; then (5) becomes

$$
y_{1}=0.72582 x_{1}-17.90196
$$

Moreover, if the actual position is $x_{2}$ and the measured position is $y_{2}$, (5) becomes

$$
y_{2}=0.72582 x_{2}-17.90196
$$

Subtracting (7) to (6) gives

$$
y_{2}-y_{1}=0.72582\left(x_{2}-x_{1}\right) .
$$

Noting $\left(x_{2}-x_{1}\right)$ is the actual displacement of micrometer $\Delta x$ and $\left(y_{2}-y_{1}\right)$ is the corresponding measured displacement $\Delta y$, so (8) becomes

$$
\Delta y=0.72582 \cdot \Delta x
$$



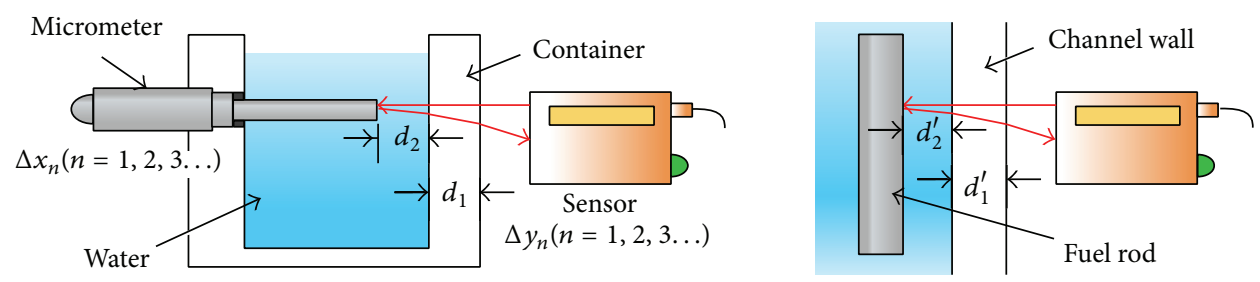

FIGURE 7: Measurement calibration device.

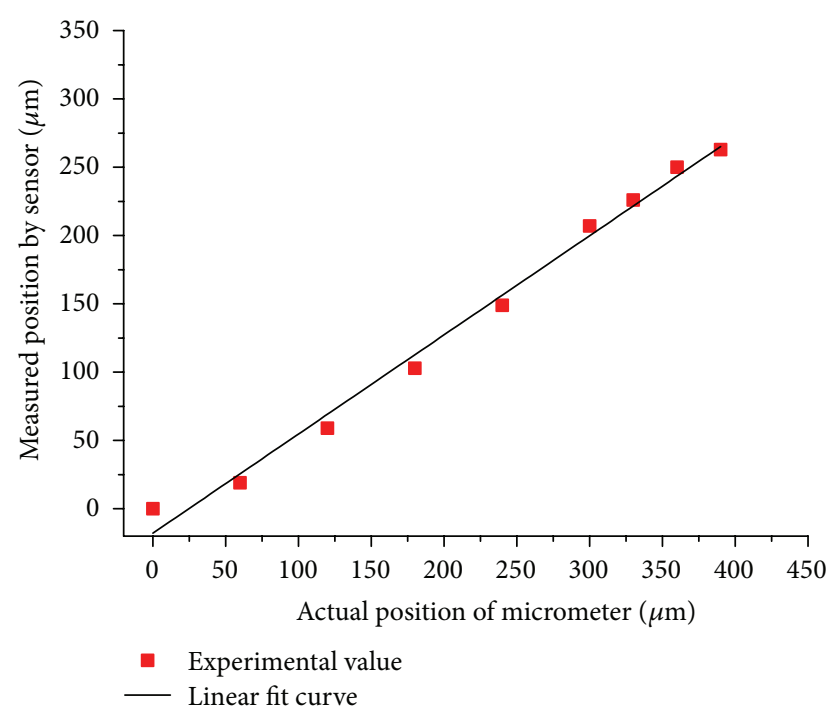

FIGURE 8: Measurement calibration curve.

From (9), it is clear that when the micrometer moves forward $1 \mu \mathrm{m}$, the corresponding measured value by displacement sensor is $0.72582 \mu \mathrm{m}$, with a deviation of $0.27418 \mu \mathrm{m}$, which is caused by the refraction. For eliminating the deviation, it is necessary to correct the measured value of laser displacement sensor according to (9).

3.3. Structure Improvement. Generally, the vibration behavior of fuel rods is random in FIV experiment, including forward motion and lateral motion. Since the fuel rods are centrosymmetric structure, statistical characteristics of the vibration in forward direction and lateral direction are the same. Thus, it is only needed to measure the forward motion during the experiment to know the vibration characteristic of fuel rods. However, when a fuel rod vibrates in both directions, as shown in Figure 9(a), the position of the laser spot projected by the sensor will change with the vibration of fuel rod, moving along the surface of cylindrical rod and causing the measured value $d_{1}$ to be larger than the actual displacement $d_{2}$. That has a substantial influence on the result. In order to solve this problem, the structure of fuel rod has been improved by fixing a tiny metal sheet on the surface of fuel rod. The size of metal sheet is shown in Figure 9(c), and one side of the sheet is flat. In this way, even though the position of laser spot still changes with the movement of fuel rod, the laser spot could move on a flat surface instead of curved surface, as shown in Figure 9(b). As a result, in this situation the measured value $d_{1}^{\prime}$ is the same as the actual displacement $d_{2}^{\prime}$.

3.4. Measuring System. The hardware of the measuring system mainly consists of a noncontact and high-speed laser displacement sensor (LTC-025-04-SA, MTI), a high-speed data acquisition card (Daq3000, Iotech), and data acquisition soft system (DASYLab, National Instruments). The measuring system is shown in Figure 10. In order to reduce the sampling signal bias and frequency alias, a low-pass filter is used to process the detected signal for preventing the highfrequency signal. In addition, a correction module is added to correct the measurement deviation caused by refraction according to (9).

\section{The Experimental Results and Analysis}

4.1. Reliability Analysis. In practice, for reducing the difficulty of FIV experiment, the experiment is always carried out at the low temperature and pressure conditions, such as FIV experiment of heat exchange tube in steam generator. In the same way, the FIV experiment of small scale fuel assembly in supercritical water loop is also carried out at the low temperature and pressure conditions. Even though the natural frequencies of fuel rods in FIV experiment are almost the same with those in the supercritical water loop, the vibration behaviors are different because of the difference between the supercritical fluid and subcritical fluid in two cases. Thus, a reliability analysis is needed to make sure the experimental result is reliable. Because the bottom ends of rods are fully constrained, the fuel rods have no displacement in the axial direction even if there is a density gradient along the rod induced by heating. Therefore, it is only needed to analyze the difference of radial displacement in two cases.

Actually, the average value of fluid exciting force $\widehat{F}$, which could cause radial displacement of fuel rod, is proportional to the density of fluid $\rho$ and the square of velocity $V$ (10). Moreover, when the size of channel is fixed, $\widehat{F}$ is proportional to the square of flow rate $Q(11)$. Consider

$$
\begin{gathered}
\widehat{F} \propto \rho \cdot V^{2} \\
\widehat{F} \propto \rho \cdot\left(\frac{Q}{s}\right)^{2} .
\end{gathered}
$$

Since the fluid used in the experiment is normal temperature water $\left(25^{\circ} \mathrm{C}\right)$, whose density is larger than that of 


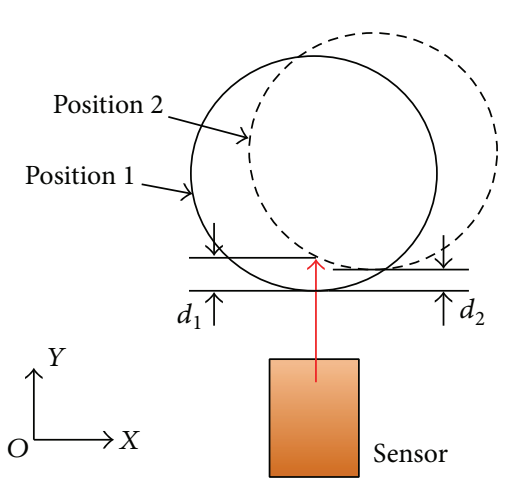

(a) Original structure $d_{1}>d_{2}$

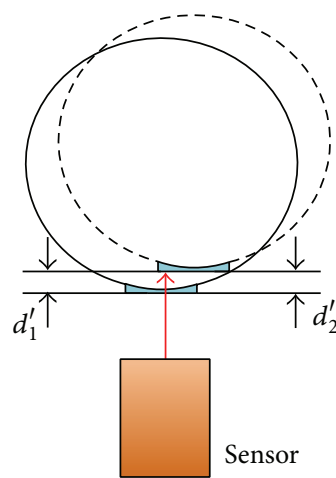

(b) Improved structure $d_{1}^{\prime}=$ $d_{2}^{\prime}$
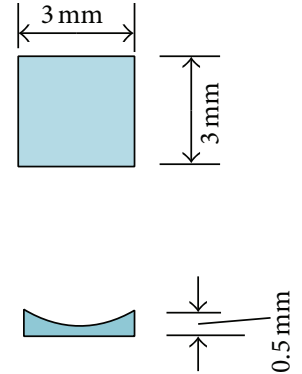

(c) Size of metal sheet

FIGURE 9: Schematic of improved structure.

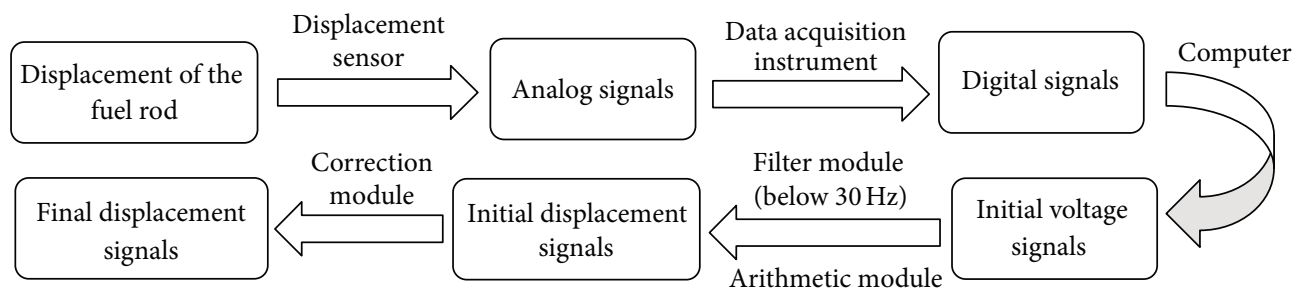

FIGURE 10: Schematic diagram of data acquisition system.

supercritical water, according to (10), it can be noted that the average value of fluid exciting force $\widehat{F}$ in the experiment is larger than that in the supercritical water loop under the same flow rate. In consequence, the average value of axial displacement in the experiment is much larger, meaning the experimental results are conservative. Therefore, if the fuel rod can meet the mechanical performance requirements in the FIV experiment, it can definitely meet the requirements of safety and reliability in the supercritical water loop.

4.2. Data Validation. For the vibration induced by turbulence and leakage flow which is random [3], an infinite record should theoretically be used to describe this process. However, only a finite length record and data can be obtained in the experiment. So as to use the characteristics of a finite length time history to characterize the whole process of random vibration, this vibration has to be ergodic [10]. So before making further analysis of the experimental data, it is necessary to make sure whether the random vibration of fuel rod is ergodic or not. Therefore, the experiment has been repeated several times under the same conditions, and the comparison among the statistical values of these experimental results is made. Table 3 shows the root mean square (rms) displacement of the fuel rod in through hole case. It can easily be found from Table 3 that the rms displacement is almost the same in these experiments under the condition of the same flow rate. That means the vibration of fuel rod is a stationary ergodic process.
4.3. Spectral Analysis. Since there is no Fourier transform for a random signal, its spectral characteristics are always characterized by the power spectral density (PSD), which is the measurement for the distribution of vibrational energy in frequency domain and contains the information about amplitude of vibration in time domain [11]. Figures 11 and 12 separately show the displacement PSD of the fuel rod in two different cases with different flow rate. It is shown in Figure 11 that, with the increase in flow rate, there are obvious changes in power spectrum curves at the frequency range of 6-20 Hz. According to the meaning of PSD mentioned above, it is certain that with the increase of flow rate, the proportion of vibrational energy distribution, and the amplitude of vibration increase at the frequency range of $6-20 \mathrm{~Hz}$. In comparison, it can be easily concluded from Figure 12 that there is no significant change in power spectrum curve under the same conditions, except that the magnitude of spectrum tends to become larger. The reason for that is that the increase of flow velocity will lead to the enhancement of turbulent-boundary-layer pressure fluctuations, which is the most important near-field noise to induce the vibration of fuel rods [12].

As shown in Figure 13, both the shape of curves and the magnitude of spectrums in two cases are essentially the same in low-frequency bands below $5 \mathrm{~Hz}$ at $q_{v}=3.62 \mathrm{~m}^{3} / \mathrm{h}$, but the trends of two curves become much different after $5 \mathrm{~Hz}$. The curve declines gradually with frequency in blind hole case. On the contrary, in the through hole case, the curve shows an ascending trend in the frequency ranging from 
TABLE 3: Root mean square displacement of the fuel rod in through hole case.

\begin{tabular}{|c|c|c|c|c|}
\hline \multirow{2}{*}{ Flow rate $\left(\mathrm{m}^{3} / \mathrm{h}\right)$} & \multicolumn{4}{|c|}{ Root mean square displacement $(\mu \mathrm{m})$} \\
\hline & 1 st & 2nd & 3 th & Average value \\
\hline 0.63 & 10.71 & 11.33 & 11.08 & 11.04 \\
\hline 1.39 & 13.17 & 13.62 & 13.74 & 13.51 \\
\hline 2.12 & 15.08 & 14.97 & 15.34 & 15.13 \\
\hline 2.88 & 17.04 & 17.22 & 16.86 & 17.03 \\
\hline 3.62 & 19.69 & 19.13 & 19.42 & 19.41 \\
\hline
\end{tabular}

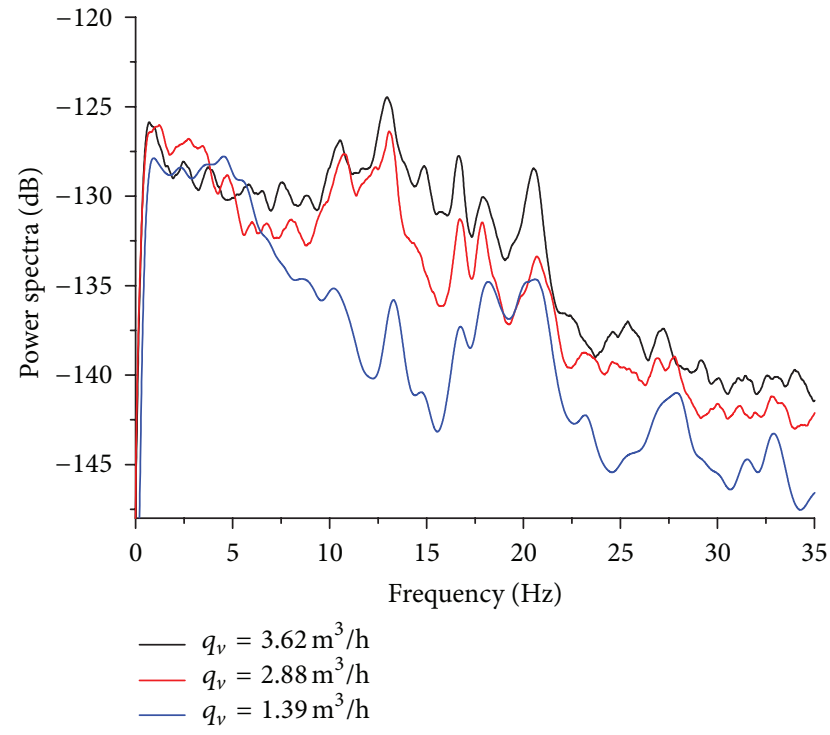

FIGURE 11: Displacement power spectral density of the fuel rod in through hole case.

$6 \mathrm{~Hz}$ to $13 \mathrm{~Hz}$ and reaches the peak value at $13 \mathrm{~Hz}$, and then the curve decreases after $13 \mathrm{~Hz}$. As a consequence, it can be concluded that the differences in PSD between these two cases are mainly caused by leakage flow occurring on the top of fuel rods. Furthermore, the influence of leakage flow includes two aspects: one is the change in the distribution of vibrational energy in spectrum, and the other is the increase in the magnitude of spectrum at the frequency range of 6-20 Hz.

Theoretically, since (1) is applicable for the studies of leakage-flow-induced vibration of the fuel rod, it can be used to calculate the vibration of fuel rod caused by leakage flow only if associated with appropriate boundary conditions. On the other hand, if the boundary conditions are not set properly, the computational result of (1) could not show the influence of leakage flow mentioned above. Therefore, the experimental results provide a benchmark for the theoretical calculation, and comparing the experimental result and numerical result could determine whether the boundary condition is proper for the studies related to leakage-flowinduced vibration.

It is shown in Figure 14 that the power spectrum curves in two cases essentially have the same changing tendency

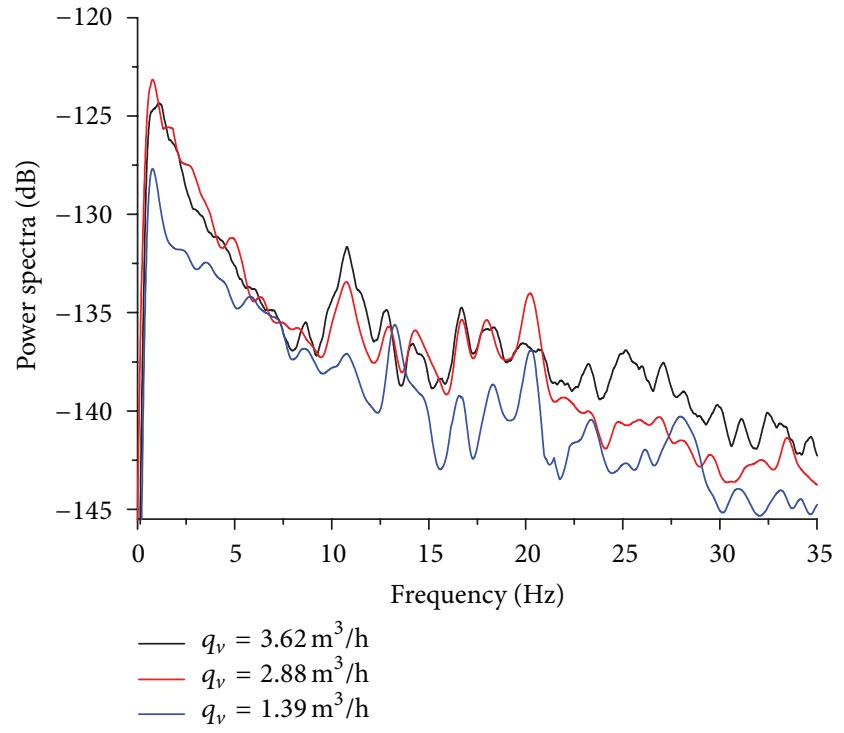

FIGURE 12: Displacement power spectral density of the fuel rod in blind hole case.

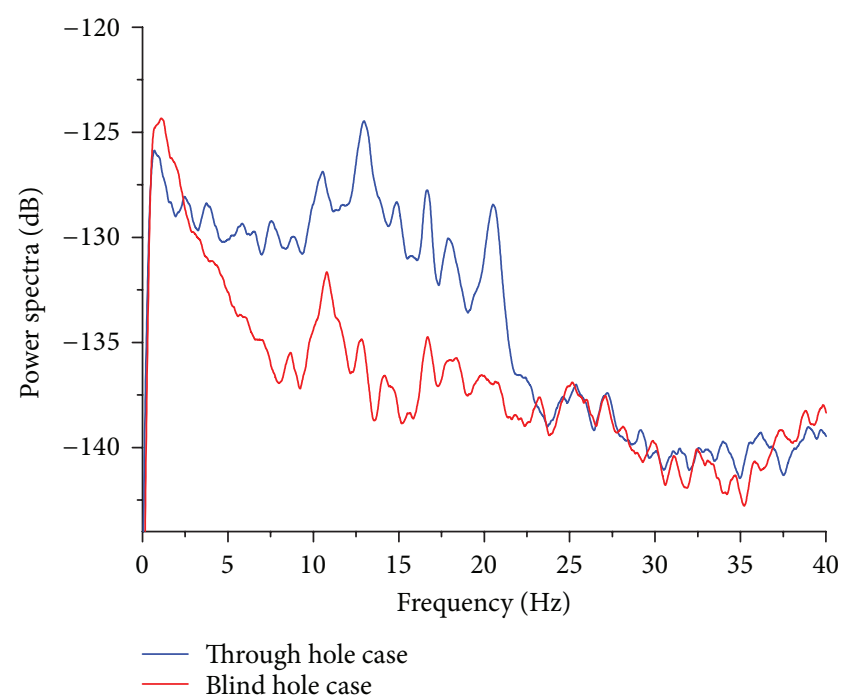

FIGURE 13: Displacement power spectral density of the fuel rod in two cases at $q_{v}=3.62 \mathrm{~m}^{3} / \mathrm{h}$. 


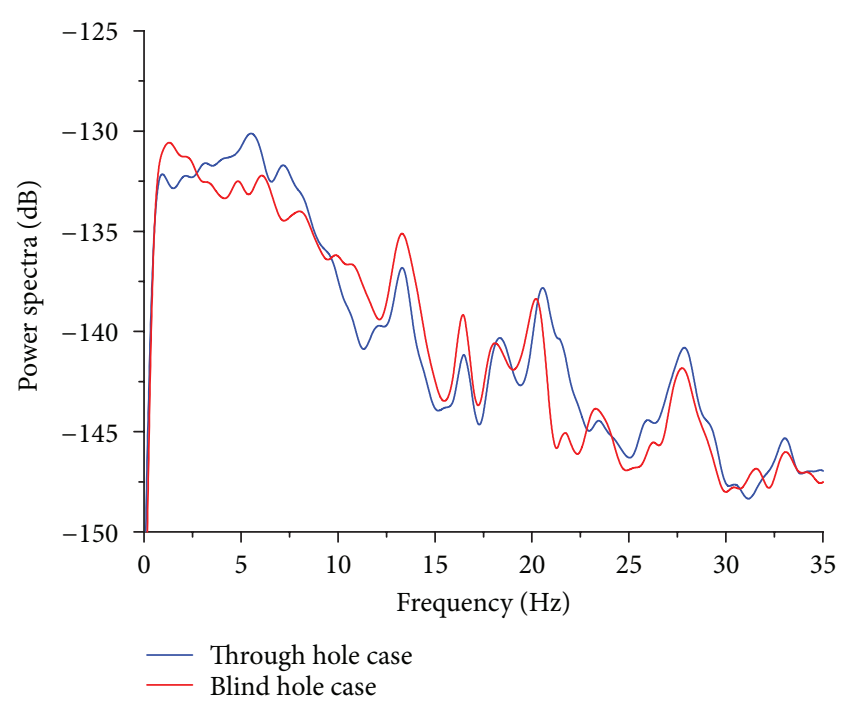

Figure 14: Displacement power spectral density of the fuel rod in two cases at $q_{v}=0.62 \mathrm{~m}^{3} / \mathrm{h}$.

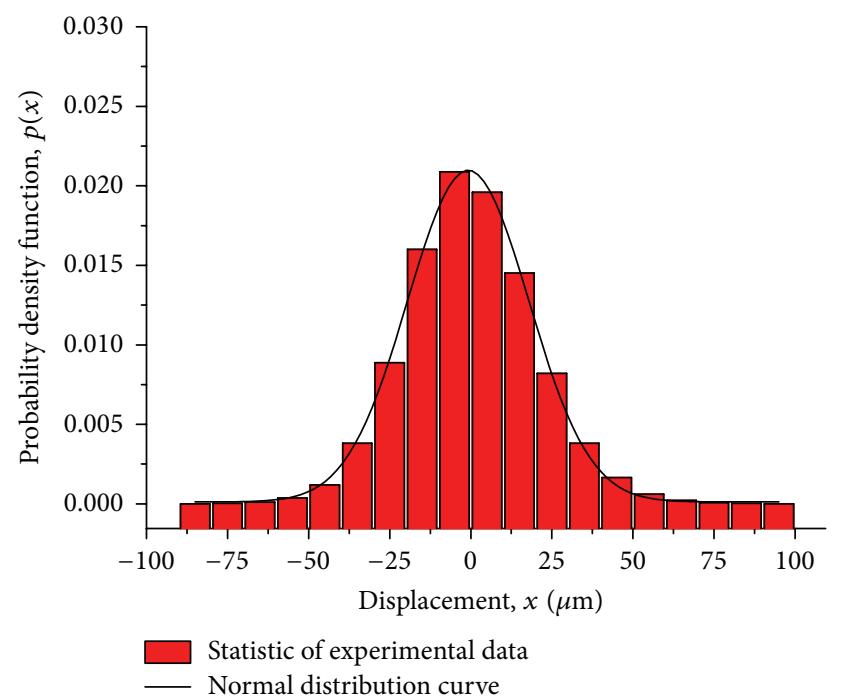

FIGURE 15: Probability density distributions of displacements in through hole case at $q_{v}=3.62 \mathrm{~m}^{3} / \mathrm{h}$.

at $q_{v}=0.62 \mathrm{~m}^{3} / \mathrm{h}$, and the reason is that the influence of leakage flow weakens as the flow rate decreases. Compared with turbulent-boundary-layer pressure fluctuations, leakage flow has little influence on the vibration of fuel rods when the flow rate reduces to a certain value.

4.4. Statistical Analysis. After making a statistical analysis on the vibration behavior of fuel rods at $q_{v}=3.62 \mathrm{~m}^{3} / \mathrm{h}$, the probability density distributions of displacements at midpoint in both cases are obtained; they all could be fitted well by a normal distribution function (see Figures 15 and 16). From the comparison, it can be found that the distribution of vibration displacements in blind hole case, in contrast to the one in through hole case, is more concentrated at $0 \mu \mathrm{m}$. On

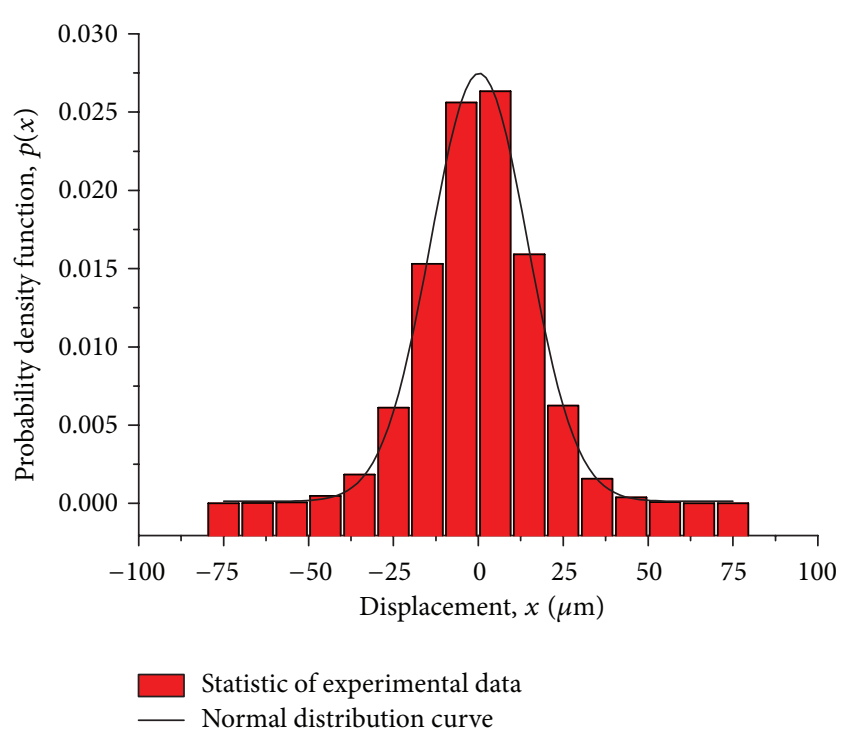

Figure 16: Probability density distributions of displacements in blind hole case at $q_{v}=3.62 \mathrm{~m}^{3} / \mathrm{h}$.

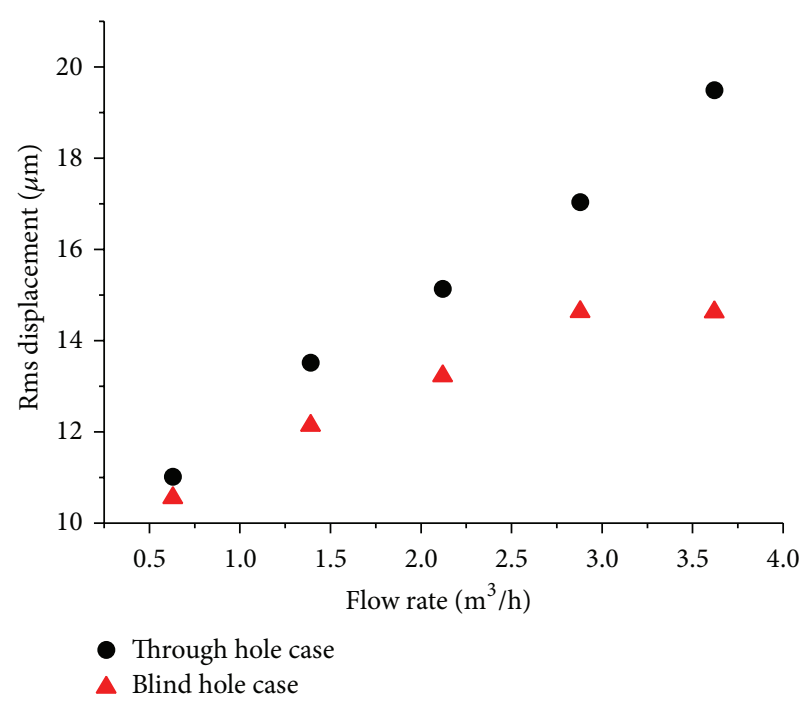

FIGURE 17: Root-mean-square displacement changing with flow rate in two cases.

the basis of probability statistics theory, it can be determined that the variance of random vibration, which represents the dynamic component of vibration amplitude, is smaller in blind hole case than that in through hole case. The reason is the existence of leakage flow in through hole case as well.

4.5. Analysis of the Root Mean Square Displacement. The absolute value of amplitude at certain time is meaningless because the vibration of fuel rod is random. Therefore, we calculate the root mean square (rms) displacement, which is a statistical measurement of the magnitude of the varying displacement and contains the information about vibration energy. The rms displacements at midpoint changing with flow rate in two cases are compared in Figure 17. It is found 


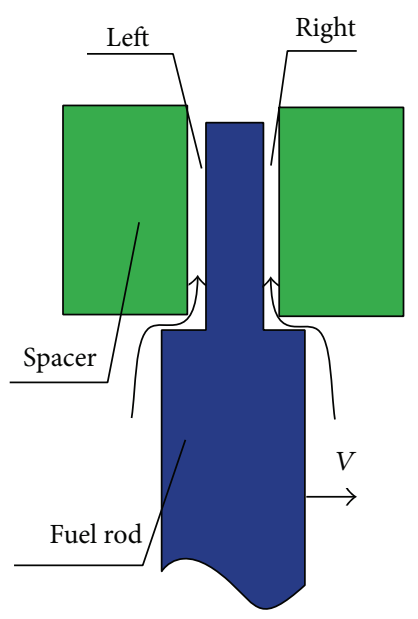

FIGURE 18: Diagram to explain the mechanism associated with leakage-flow-induced instability.

that the uptrend of rms displacement in through hole case is more obvious than that in blind hole case. In through hole case, if the rod has a small rightward velocity $V$ caused by turbulence, it will lead to the reduction of right side clearance (see Figure 18). As a result, the flow across the right side clearance will decrease with time. The opposite situation exists with respect to the flow in the left side clearance. Because of flow velocity redistribution in the clearance, there are changes in the static pressure on both sides of clearance consequently. It gives rise to a net fluid force acting on the top end of fuel rod in the same direction as the motion. Hence, this results in amplified motion. Under the same other conditions, the difference between rms displacements in two different cases is strongly dependent on leakage flow. Therefore, in order to reduce the flow-induced vibration, which may lead to fatigue of the fuel rods, it is more reasonable to use the spacers with blind hole to assemble the small scale fuel rods in the supercritical water loop.

\section{Conclusions}

An experiment is made to investigate flow-induced vibration of rods exposed to an axial flow within a rigid square channel, especially the vibration caused by leakage flow. In order to improve the experimental accuracy, measurement calibration is performed to correct the measurement deviation caused by refraction before the FIV experiment. According to the experimental data and analytical results, three useful conclusions are obtained as follows.

(1) When flow rate is low, the vibration of rods is mainly caused by turbulence flow, and leakage flow has little influence on that.

(2) As flow rate increases, the influence of leakage flow becomes obvious, which could change the distribution of vibrational energy in spectrum, increasing the vibrational energy in high-frequency band. Furthermore, it could be treated as a benchmark for the theoretical studies to validate the applicability of boundary condition set for leakage flow. If a boundary condition is set properly, the result of theoretical calculation would show the influence of leakage flow found in the experiment; otherwise this boundary condition is not set properly.

(3) Under the same other conditions, the difference between rms displacements in two different cases is strongly dependent on leakage flow. Therefore, from the point of view of structure safety and reliability, it is more reasonable to improve the design by using the spacers with blind hole to assemble the small scale fuel rods in the supercritical water loop.

\section{Conflict of Interests}

The authors declare that there is no conflict of interests regarding the publication of this paper.

\section{References}

[1] R. D. Blevins, Flow-Induced Vibration, Van Nostrand Reinhold Company, 1977.

[2] D. E. Hobson, "Fluid-elastic instabilities caused by flow in an annulus," in Proceedings of 3rd International Conference on Vibration in Nuclear Plant, pp. 421-428, Keswick, UK, 1982.

[3] S. S. Chen, Flow-Induced Vibration of Circular Cylindrical Structures, Springer, Berlin, Germany, 1987.

[4] M. P. Païdoussis, "A review of flow-induced vibrations in reactors and reactor components," Journal Nuclear Engineering and Design, vol. 74, no. 1, pp. 31-60, 1983.

[5] D. Mateescu and M. P. Paidoussis, "Unsteady potential flow in an axially variable annulus and its effect on the dynamics of the oscillating rigid center-body," Journal of Fluids Engineering, Transactions of the ASME, vol. 107, no. 3, pp. 421-427, 1985.

[6] M. Arai and K. Tajima, "Leakage-flow-induced vibrations of an axisymmetric body: part 1-analysis of the moment acting on an axisymmetric body for rotational motion," JSME International Journal C, vol. 41, no. 3, pp. 347-354, 1998.

[7] D.-W. Li, S. Kaneko, and S. Hayama, "A study on annular leakage-flow-induced vibrations," Journal of Fluids and Structures, vol. 16, no. 7, pp. 909-930, 2002.

[8] M. A. Langthjem, H. Morita, T. Nakamura, and M. Nakano, "A flexible rod in annular leakage flow: influence of turbulence and equilibrium offset, and analysis of instability mechanisms," Journal of Fluids and Structures, vol. 22, no. 5, pp. 617-645, 2006.

[9] K. Fujita, H. Morikazu, and A. Shintani, "A consideration on pre- and post-instability of an axisymmetric elastic beam subjected to axial leakage flow," Journal of Fluids and Structures, vol. 23, no. 3, pp. 463-478, 2007.

[10] D. E. Newland, An Introduction to Random Vibrations and Spectral Analysis, China Machine Press, Beijing, China, 1984.

[11] Z. Q. Hu, Q. Y. Fa et al., Technique of Random Vibration Test, Chinese Metrology Press, Beijing, China, 1984.

[12] S. S. Chen, "Response of a flexible rod to near-field flow rate," in Proceedings of the Conference on Flow-Induced Vibrations in Reactor System Components (ANL-7685 '70), pp. 5-31, Argonne National Laboratory, Argonne, Ill, USA, 1970. 


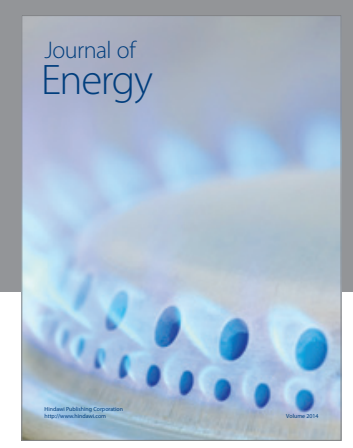

Journal of

Industrial Engineering
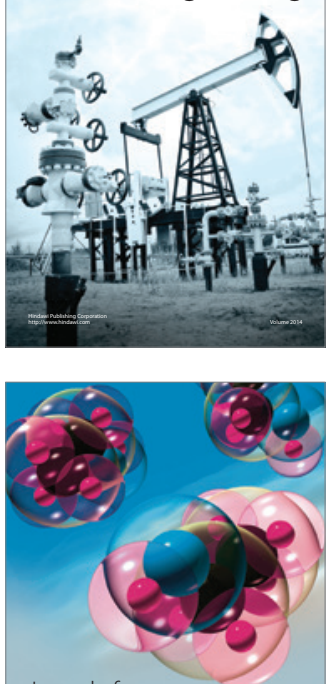

Fuels
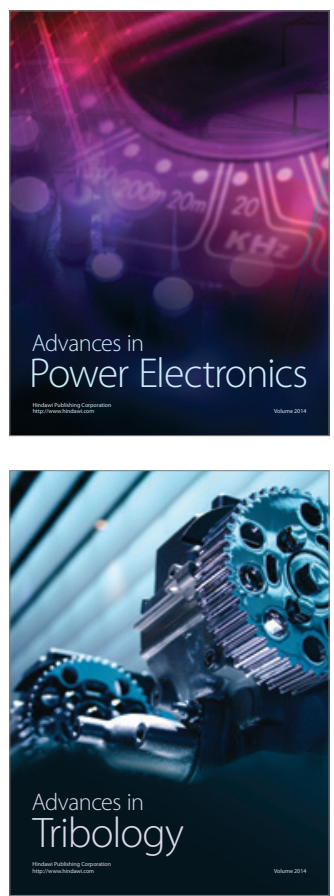

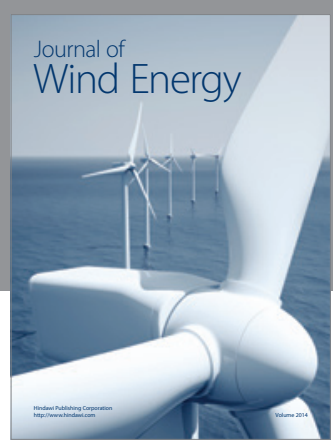

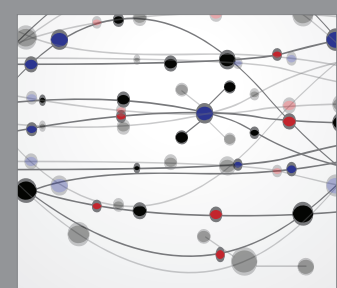

The Scientific World Journal

Submit your manuscripts at http://www.hindawi.com

Journal of

Structures
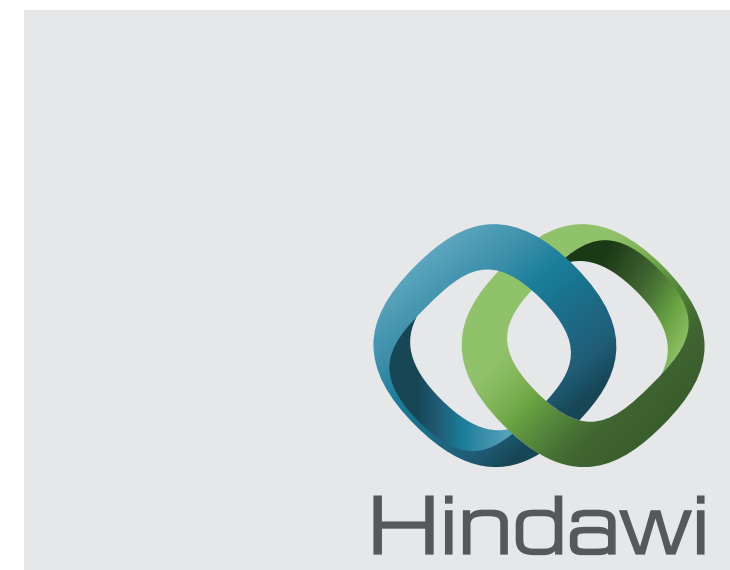

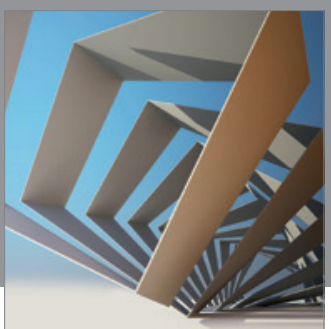

Rotating

Machinery
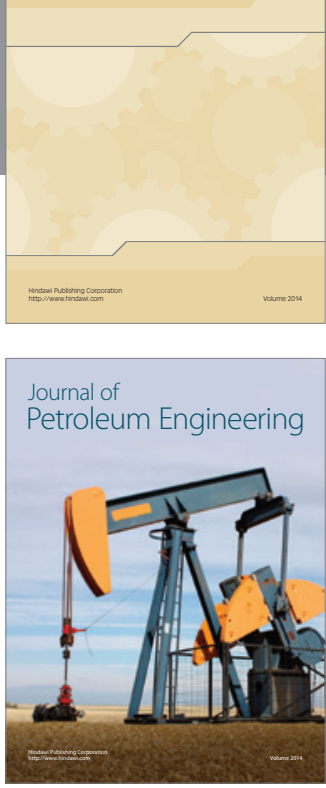

Journal of

Solar Energy
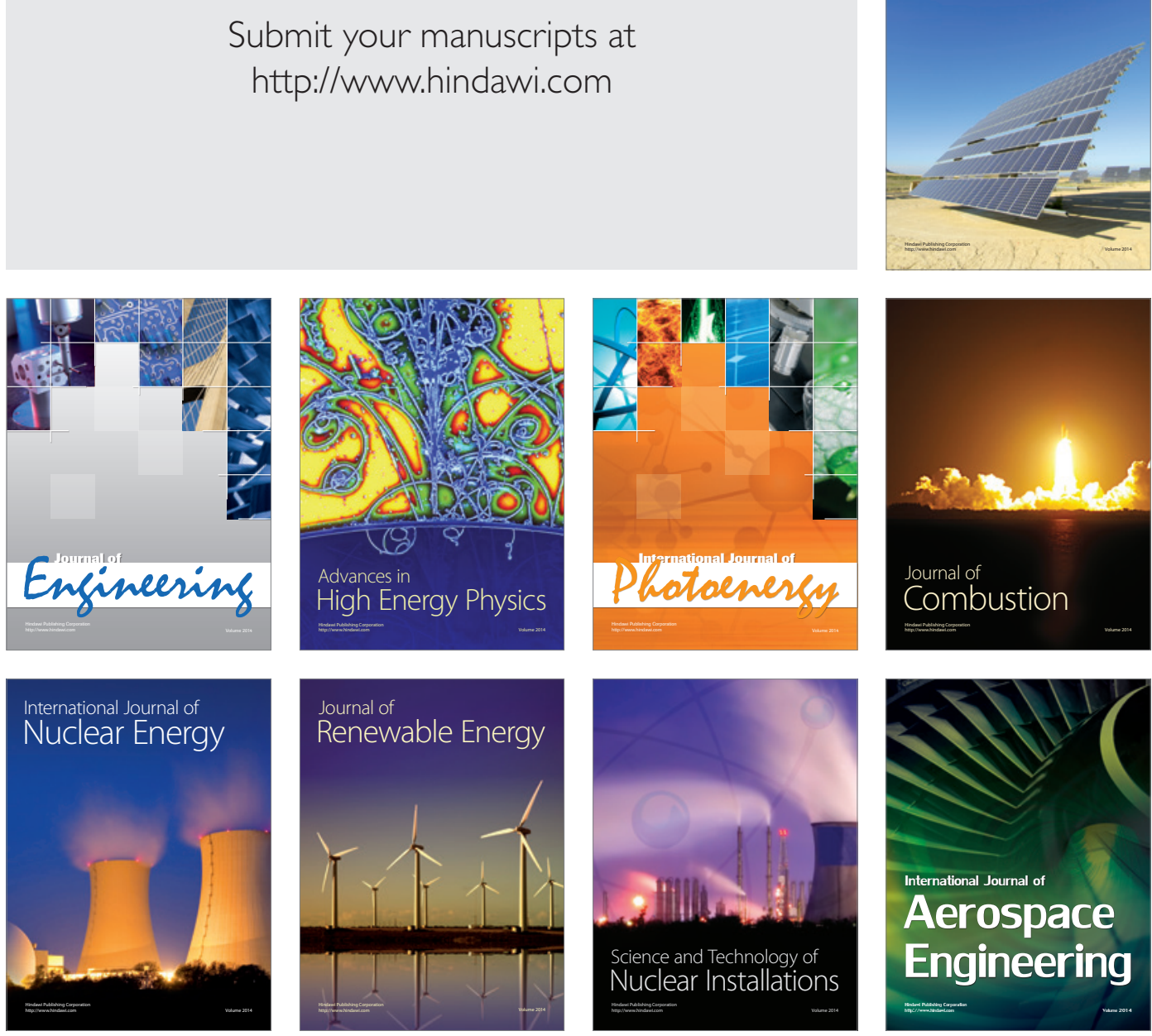